\title{
Postnatal Changes in the Distribution of Succinate Dehydrogenase Activities among Diaphragm Muscle Fibers
}

\author{
GARY C. SIECK AND CESAR E. BLANCO \\ Departments of Anesthesiology and Physiology and Biophysics, Mayo Clinic and Mayo Foundation, \\ Rochester, Minnesota 55905
}

\begin{abstract}
The distributions of oxidative capacities among type-identified muscle fibers in the developing cat diaphragm were examined by quantifying succinate dehydrogenase (SDH) activity using a microdensitometric procedure. Animals were studied during the first six weeks of postnatal development and compared to adults. Muscle fiber SDH activities were initially low during the first 2 postnatal wk, then increased to their highest values between 3 and 6 wk. Thereafter, fiber SDH activities declined to adult values. At each age, the distributions of SDH activities for both type I and II fibers were unimodal. Thus, no objective basis exists for subclassifying type II fibers based on differences in oxidative capacity. Fibers could be subclassified as type IIA, IIB, or IIC based on the acid pH lability of ATPase staining. In neonates, approximately $90 \%$ of all fibers were classified as type IIC. Thereafter, the proportion of IIC fibers decreased while the proportions of type I, IIA, and IIB increased. Adult fiber type proportions were reached by 6 wk of age. The SDH activity of type I fibers was generally higher than that of type II fibers at all ages, although there was considerable overlap in the distributions of SDH activities among type I and II fibers. The SDH activity of type IIC fibers was also higher than that of either type IIA or IIB during development. Only in the adult diaphragm was the SDH activity of type IIA fibers higher than that of type IIB. At no age could type IIA, IIB, or IIC fibers be discriminated based solely on differences in oxidative capacity. (Pediatr Res 29: 586593,1991 )
\end{abstract}

\section{Abbreviations}

SDH, succinate dehydrogenase

NADH-TR, NADH-tetrazolium reductase

NBT, nitroblue tetrazolium

NBT-dfz, nitroblue tetrazolium diformazan

CSA, cross-sectional area

$\mathrm{CV}$, coefficient of variation

IQR, interquartile range

Mhc, myosin heavy chain

Diaphragm muscle fibers have been classified into different types based upon differences in histochemical staining patterns

Received October 23, 1990; accepted January 21, 1991

Correspondence: Gary C. Sieck, Ph.D., Division of Anesthesia Research, Mayo Clinic, Rochester, MN 55905.

Supported by grants from the NIH Heart, Lung, and Blood Institute (HL 37680 and HL 34817).

Current address: Department of Anatomy and Cell Biology, UCLA School of Medicine, Los Angeles, CA 90024.
(1-9). Fibers staining lightly for myofibrillar ATPase after alkaline preincubation have been classified as type I and those staining darkly for ATPase as type II. Type II (fast-twitch) fibers have been further subclassified using one of two major schemes. In the procedures established by Brooke and Kaiser $(10,11)$, type II fibers were subclassified as IIA, IIB, or IIC based upon differences in the acid $\mathrm{pH}$ lability of staining for ATPase. In the procedure devised by Peter et al. (12), fast-twitch fibers were subclassified as fast-twitch glycolytic or fast-twitch, oxidative glycolytic based upon differences in staining intensity for oxidative enzymes.

It should be noted that the subclassification of type II fibers as fast-twitch glycolytic or fast-twitch, oxidative glycolytic is highly subjective, depending upon perceived differences in the relative staining intensity of fibers for oxidative enzymes (12). In recent studies $(6,7)$, we quantified the activity of the mitochondrial oxidative enzyme SDH in muscle fibers of the adult cat diaphragm. We found that the distribution of SDH activities for type II fibers was unimodal, indicating that in the adult muscle, no clear objective threshold exists for subclassifying fibers based upon oxidative capacity.

In developing skeletal muscles, the subclassification of type II fibers based upon differences in oxidative enzyme staining may be even more illusive because of the reported uniformity of enzyme staining (13-17). Yet, in the developing diaphragm, muscle fibers have still been classified based on perceived differences in staining for oxidative enzymes, with conflicting results. For example, Keens et al. $(1,2)$ reported that, in the neonatal human diaphragm, all of the approximately $75 \%$ type II fibers displayed low staining intensity for NADH-TR. In contrast, Maxwell et al. (3) reported that in the neonatal baboon diaphragm, all of the approximately $60 \%$ type II fibers had high staining intensity for NADH-TR. The purpose of our study was to quantify the SDH activities of muscle fibers in the cat diaphragm at different ages during postnatal development.

\section{MATERIALS AND METHODS}

Animals. Twenty-five cats were studied at varying ages during postnatal development $(0-3 \mathrm{~d}$ of age, termed $1 \mathrm{st} \mathrm{wk}, n=5 ; 14-$ 15 days of age, termed 2 nd wk, $n=5 ; 21-22$ d of age, termed 3rd wk, $n=6 ; 42-43 \mathrm{~d}$ of age, termed 6 th wk, $n=5$; and adults of either sex, $n=5$ ). All younger animals were obtained from a breeding colony consisting of three females and one male. Litter size averaged 5.0 $\pm 1.6(\mathrm{SD})$ animals and, usually, three animals per litter were used for these studies, but not within a single age group. Animal body weights were measured daily, and animals with median body weights for a given litter were selected for study. Exposure of the female to the male was limited so that gestational period could be determined; gestational age ranged from 62 to $75 \mathrm{~d}$ with an average of $67 \pm 4 \mathrm{~d}$. Animals were grouped according to postnatal ages. The postnatal ages of the 
animals at the time of study were selected to reflect the entire period of preweaning development. One caveat in evaluating these results is that the interval between age groups was unequal although the terms 1st, 2nd, 3rd, and 6th wk were used.

Muscle histochemistry. Animals were killed with an overdose of pentobarbital sodium injected intraperitoneally. The diaphragm was excised and muscle samples from the mid-costal region were quickly frozen in isopentane cooled to its melting point by liquid nitrogen. Muscle fibers were frozen at their excised length, not at optimal fiber length. In other studies, we have determined that excised fiber length is approximately $70 \%$ of optimal fiber length (unpublished observations). The measured CSA at this shorter fiber length is increased proportionately, but measurements of fiber SDH activity are unaffected because they are normalized for fiber volume (unpublished observations). Serial cross-sections of muscle fibers were cut at $10 \mu \mathrm{m}$ thickness using a cryostat (model no. 2800E; Reichert-Jung, Buffalo, NY) kept at $-20^{\circ} \mathrm{C}$. The motorized drive of this cryostat permitted sections to be cut with an error of less than $0.2 \mu \mathrm{m}$ in thickness. This is especially important in quantitative histochemistry, where path length for light absorbance is critical (see below).

Sections were stained for ATPase after alkaline preincubation ( $\mathrm{pH} 9.0$, for $9 \mathrm{~min}$ at $25^{\circ} \mathrm{C}$ ) to broadly classify fibers as type I or II (10-12). Alternate sections were also stained for ATPase after acid preincubation at $\mathrm{pH} 4.3$ and 4.6 (for $5 \mathrm{~min}$ at $25^{\circ} \mathrm{C}$ ) to subclassify fibers as type IIA, IIB, or IIC $(10,11)$. Differences in staining intensities for ATPase were quantified using microdensitometric analysis implemented on an image processing system (see below) (compare 6, 7).

Quantification of fiber SDH activity. The SDH activities of the classified muscle fibers were determined in alternate serial sections using a microdensitometric procedure that has been previously described in detail $(6,7,18)$. Briefly, the concentrations of enzymatic substrate (succinate; $48 \mathrm{mM}$ ), electron carrier (e.g. 1methoxy-phenazine methosulfate; $1.0 \mathrm{mM}$ ), electron transport chain inhibitor (sodium azide; $0.75 \mathrm{mM}$ ), and $\mathrm{pH}$ (7.6) of the $\mathrm{SDH}$ reaction medium are optimized for measuring the maximum SDH reaction rate (18).

With the conversion of succinate to fumarate in the $\mathrm{SDH}$ reaction, the $\mathrm{H}^{+}$ions released are used to reduce NBT $(1.5 \mathrm{mM}$ in the reaction medium) to an insoluble colored compound (NBT-dfz). The concentration of NBT-dfz deposited within a fiber is directly proportional to the amount of fumarate produced by the SDH reaction ([fumarate] $=2[\mathrm{NBT}-\mathrm{dfz}]$ ) and, across a period of time, the deposition of this reaction product is a direct measure of SDH activity (expressed as mmol fumarate/L tissue/ min). The concentration of NBT-dfz can be determined from OD measurements using the Beer Lambert equation:

$$
[\mathrm{NBT}-\mathrm{dfz}]=\frac{\mathrm{OD}}{\mathrm{k} \times \mathrm{L}}
$$

where $O D$ is measured at $570 \mathrm{~nm}$ (peak absorbance wavelength of NBT-dfz), $\mathrm{k}$ is the molar extinction coefficient of NBT-dfz (38 $\left.152 \mathrm{~mol}^{-1} \cdot \mathrm{cm}^{-1}\right)$ and $\mathrm{L}$, is the light path length $(10-\mu \mathrm{m}$ section thickness).

To control the path length for light absorbance, muscle crosssections were cut at a constant thickness of $10 \mu \mathrm{m}$ (with less than $2 \%$ error). The muscle cross-sections were magnified using a light microscope. The wavelength of the transmitted light of the microscope was restricted to $570 \mathrm{~nm}$ by interposing an interference filter (peak wavelength $=570 \pm 5 \mathrm{~nm}$; Spectrofilm, Winchester, MA) between the light source (halogen lamp) and the microscope stage. A video scanner was attached to the microscope, and video images of the magnified muscle cross-sections were digitized into an array of $1024 \times 1024$ picture elements (pixels) using an image processing system (Megavision 1024XM; Megavision, Goleta, CA). The 256 possible gray levels of the video scanner were converted to OD units by calibrating against a series of neutral density filters $(0.04$ to 1.20 OD units; MellesGriot, Irvine, CA).

Video images of the muscle cross-section were digitized every $30 \mathrm{~s}$ as the SDH reaction developed. In digitizing each image, an average of 16 separate scans was calculated to reduce electronic noise. In addition, the digitized image was shade-corrected to reduce errors introduced by an uneven illumination of the image field. In both adult and younger animals, the linearity of the SDH reaction in diaphragm fibers was verified across a 10 -min period. In sections where the same fibers were reacted for $\mathrm{SDH}$ in the absence of succinate (nonspecific reduction of NBT, i.e. tissue blanks), the mean OD remained relatively constant across the entire incubation period. Because both the specific (with substrate) and nonspecific (without substrate) SDH reactions were linear for a finite period and their OD values intersected at $t=0$, the use of a single 8-min end-point reaction was justified. The OD values in the tissue blanks were subtracted from the OD values of the same fibers in sections reacted with succinate.

For each diaphragm, seven serial sections were digitized: three sections were stained for ATPase after alkaline or acid preincubation to determine fiber type; two sections were stained for $\mathrm{SDH}$ with succinate added to the incubation medium; and two sections were stained for SDH with succinate deleted from the incubation medium (i.e. tissue blanks controlling for nonspecific reduction of NBT). From each digitized image, the boundaries of approximately 125 individual fibers were outlined. A total of 250 to 500 fibers per muscle were analyzed. The mean OD of all pixels within each outlined fiber was calculated.

Fiber morphometry. The projected CSA of each pixel was also calculated by calibration with a microscope stage micrometer. The CSA of each fiber was then calculated from the number of pixels within the outlined region.

Biochemical determination of muscle SDH activity. The SDH activity of each diaphragm was also determined biochemically using a modification (19) of the method described by Pennington (20). In this modified procedure, $1 \mathrm{mmol}$ sodium azide was included in the incubation medium to inhibit cytochrome oxidase. This substitution was made so that the biochemical reaction for SDH approximated the histochemical reaction. Biochemical measurements of muscle SDH activity were standardized to protein concentration measured using the method described by Bradford (21). In contrast, histochemical determinations of fiber $\mathrm{SDH}$ activity were standardized to tissue volume.

Statistical analysis. All values are reported as means $\pm 1 \mathrm{SD}$ To standardize fiber SDH and CSA measurements across animals, the values were transformed based on the following equation:

$$
\text { Z-score }=[\mathrm{Xi}-\mathrm{u}] / \mathrm{s}
$$

where $\mathrm{Xi}$ is the SDH activity of each fiber, $\mathrm{u}$ is the mean SDH activity of all fibers within a diaphragm, and $\mathrm{s}$ is the SD of the mean.

This linear transformation yields a distribution of SDH activities and CSA for a single diaphragm in which the population mean for the muscle equals 0 (22). The distribution patterns of fiber SDH activities and CSA within a muscle are unaffected by this normalization procedure. The Z-scores across animals within an age group were then averaged. Statistical comparisons were based on a two-way (fiber type and age) analysis of variance for repeated measures and, where significant interactions between grouping factors were detected, a Mann-Whitney test was also performed. The distribution of fiber SDH activities or CSA was compared to an "expected" randomized distribution by $\chi^{2}$ analysis $(22,23)$. Nonunimodal distributions were indicated by significant deviations from the expected distribution.

\section{RESULTS}

Body weight. There was considerable variance in birth weights and weight gain across litters. This variance was controlled by 
selecting animals from different litters to represent each age group studied. Postnatal age was used to reference the data across animals instead of body weight or other measures of growth. It was observed, however, that the mean body weight of the animals increased progressively with age $(p<0.01$; Fig. 1$)$.

Muscle fiber type proportions. Muscle fiber types could be discriminated based on quantitative differences in staining intensity for ATPase after alkaline and acid preincubations $(4,5)$. Type I fibers stained lightly (OD 0.25-0.33) for ATPase after alkaline preincubation, whereas type II fibers stained dark (OD 0.75-0.81). After preincubation at $\mathrm{pH} 4.3$, type I and IIC fibers stained dark (OD 0.61-0.71), type IIA fibers stained lighter (OD $0.30-0.32$ ), and type IIB fibers stained intermediate (OD $0.46-$ 0.48 ). This difference in ATPase staining intensity was also observed after preincubation at $\mathrm{pH} 4.6$ (type I and IIC fiber OD $0.77-0.81$; type IIA fiber OD $0.62-0.63$; and type IIB fiber OD $0.0 .71-0.72$ ). It is important to note, however, that ATPase staining intensities for diaphragm fibers varied with preincubation time and temperature $(4,5)$. Therefore, these staining intensities were observed for a specific preincubation time (i.e. $5 \mathrm{~min}$ for acid preincubation and $8 \mathrm{~min}$ for alkaline preincubation) and for a specific incubation temperature (i.e. $25^{\circ} \mathrm{C}$ ).

The changing proportions of different fiber types in the cat diaphragm during postnatal development have been reported in previous papers $(4,24)$. Figure 2 summarizes these results. The proportion of type I fibers in the diaphragm increased progressively from approximately $10 \%$ at birth to $38 \%$ in the adult ( $p$ $<0.01$ ). By the 6 th postnatal wk, the proportions of type I and II fibers were comparable to those found in the adult diaphragm. In the 1st postnatal wk, all type II fibers were classified as IIC. Thereafter, the proportion of IIC fibers decreased until, by the 6 th wk, less than $2 \%$ of all fibers were classified as IIC $(p<0.01)$ The proportion of fibers classified as IIA increased abruptly at the 2 nd postnatal wk to approximately $45 \%(p<0.01)$, and then decreased to adult values of about $28 \%(p<0.01)$. The proportion of fibers classified as IIB increased progressively throughout postnatal development from $17 \%$ during the $2 \mathrm{nd}$ wh to $34 \%$ in the adult diaphragm $(p<0.01)$

Fiber SDH activity. The distributions of SDH activities for both type I and II fibers did not vary from an expected randomized distribution (i.e. they were unimodal), although the pattern of distribution varied across animals even within the same age group. The distributions of relative SDH activities (Z-scores) for type I and II diaphragm fibers from each age group were also found to be unimodal (Fig. 3). At each age, the distribution of $\mathrm{SDH}$ activities for type I fibers tended to be skewed toward

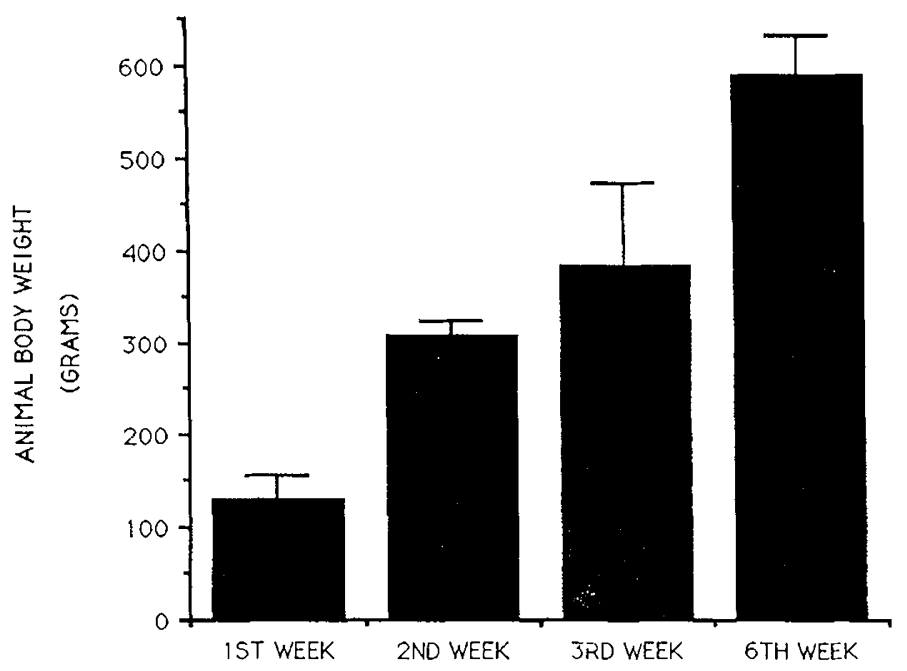

$A G E$

Fig. 1. Animal body weights increased progressively during the first 6 wk of postnatal development. Values are means \pm SD.

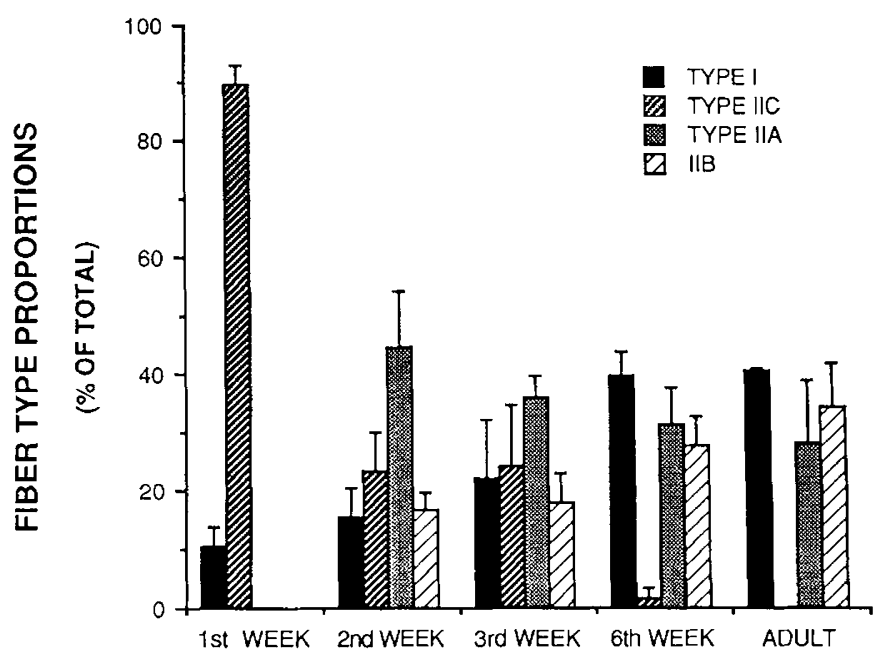

Fig. 2. Summary of the changes in fiber type proportions during postnatal development. Values are means \pm SD.

higher activities (i.e. positive Z-scores), whereas the relative SDH activities of type II fibers were lower (i.e. negative Z-scores). At each age, the distributions of SDH activities for type I and II fibers overlapped extensively (Figs. 3-5). However, a significant difference in the mean SDH activity between type I and II fibers was not apparent until after the 3rd postnatal wk $(p<0.01$; Fig. 6).

The distributions of SDH activities among type IIA, IIB, and IIC fibers also overlapped extensively (Figs. 4 and 5). During the 3rd postnatal wk, the mean SDH activity of type IIC fibers was higher than that of either type IIA or IIB fibers $(p<0.01)$ and comparable to that of type I fibers (Fig. 6). There were no significant differences in the mean SDH activity of type IIA and IIB fibers throughout the first 6 wk of postnatal development. In contrast, the mean SDH activity of type IIA fibers was significantly higher than that of type IIB fibers in the adult diaphragm $(p<0.01$; Fig. 6). Despite these differences in mean SDH activities, type IIA, IIB, or IIC fibers could not be discriminated from each other based solely on their oxidative capacity (Figs. 4 and 5).

As we previously reported $(4,24)$, the mean SDH activity of both type I and II fibers was low during the first 2 wk compared to later postnatal stages (Fig. 6). During the 3rd postnatal wk, fiber SDH activities increased 2-fold and remained high through the 6th wk of age before decreasing to adult values (Fig. 6). Biochemical determination of diaphragm SDH activity showed the same developmental trend (Fig. 7).

The $\mathrm{CV}$ of SDH activities among diaphragm fibers increased during postnatal development from $21.9 \pm 0.5 \%$ in the 1 st wk to $37.7 \pm 5.3 \%$ in the 6 th wk $(p<0.01)$. This compares to a CV in SDH activity of $42.1 \pm 3.5 \%$ among adult diaphragm fibers. The IQR (i.e. the range around the median comprising $50 \%$ of all values) of SDH activities was comparable during the first 2 postnatal wk (e.g. $0.61 \pm 0.15$ versus $0.64 \pm 0.24 \mathrm{mmol}$ fumarate/L tissue/min, respectively) and then more than doubled by the $3 \mathrm{rd}$ wk $(1.48 \pm 0.22 \mathrm{mmol}$ fumarate $/ \mathrm{L}$ tissue $/ \mathrm{min} ; p<0.01)$. Thereafter, the IQR of SDH activities remained approximately the same, despite the decrease in mean SDH activity in the adult muscle (e.g. adult $\mathrm{IQR}=1.58 \pm 0.14 \mathrm{mmol}$ fumarate $/ \mathrm{L}$ tissue $/$ $\min )$.

Fiber cross-sectional area. Fiber CSA increased progressively with age $(p<0.01$; Fig. 8). The average CSA of type II fibers increased to a greater extent during postnatal development than did that of type I fibers $(p<0.05)$. In the 1st postnatal wk, type I fibers were slightly larger than type II fibers $(p<0.05)$. From the 2 nd to 6 th wk of age, fiber CSA of type I and II fibers were comparable. In the adult, type II fibers in the diaphragm were approximately $40 \%$ larger than type I fibers $(p<0.01$; Fig. 8$)$. 
A

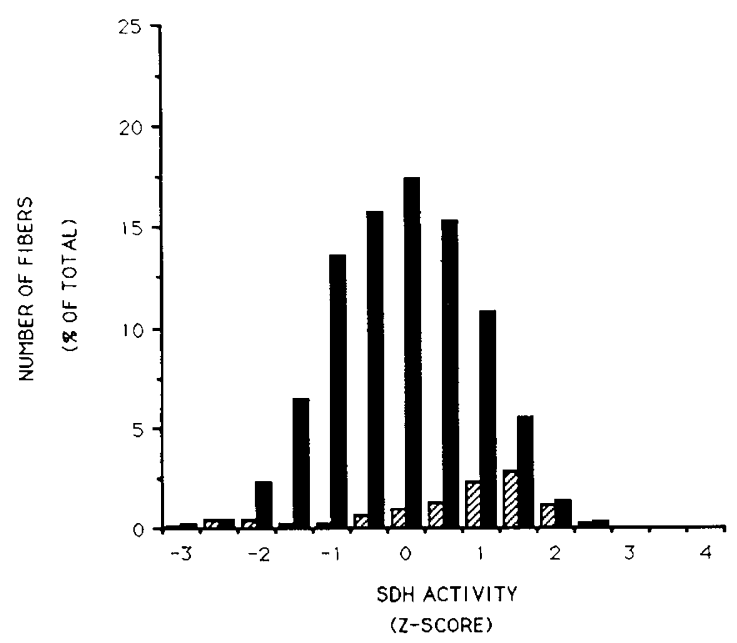

C

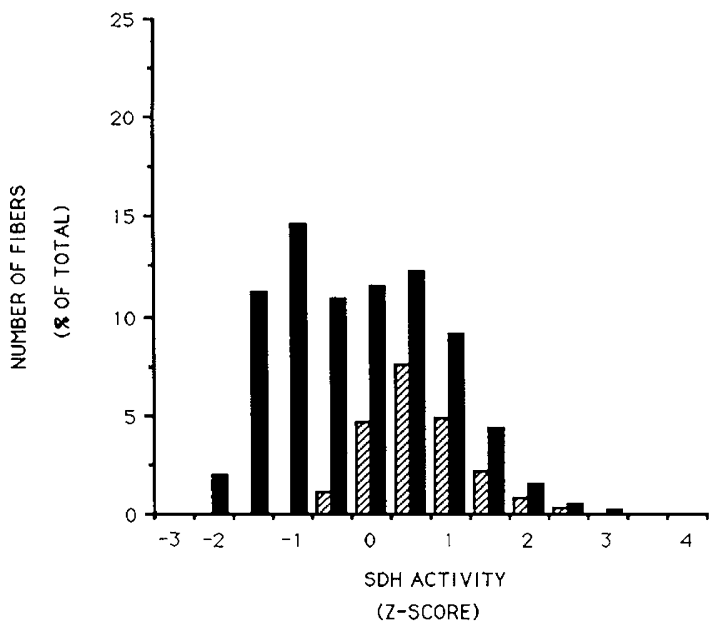

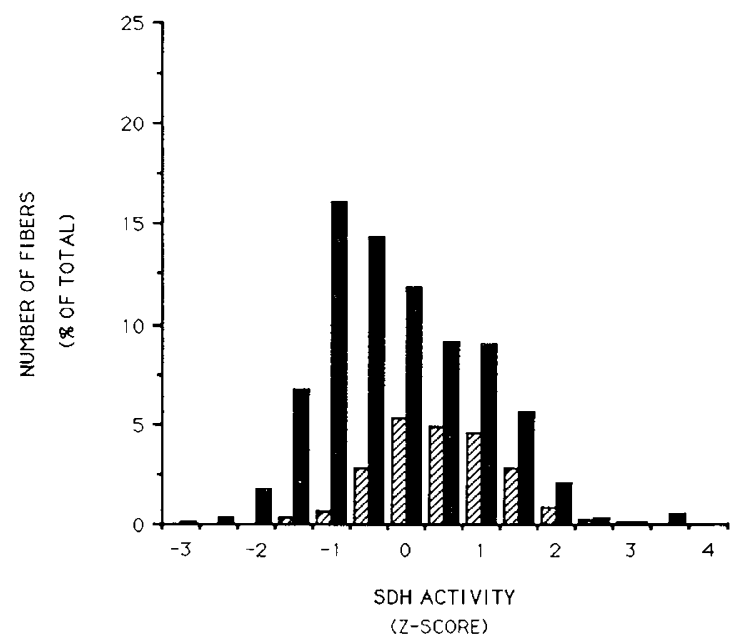

D

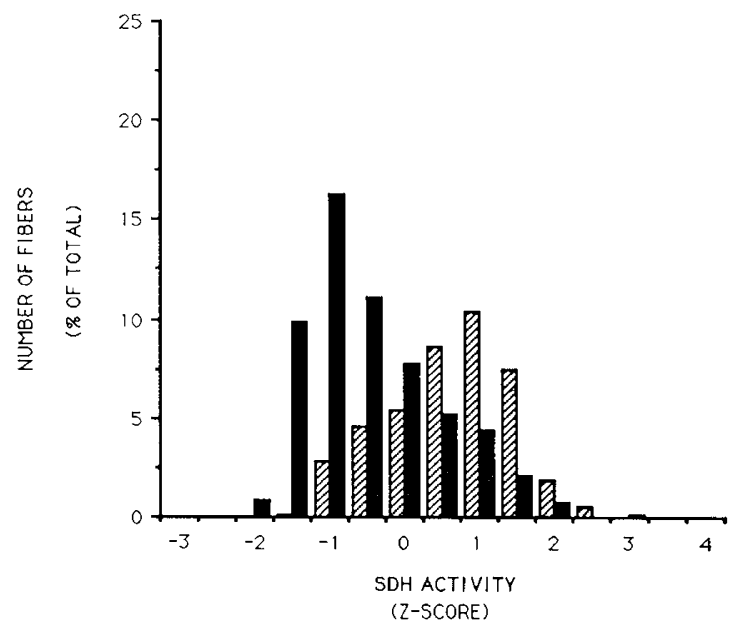

$E$

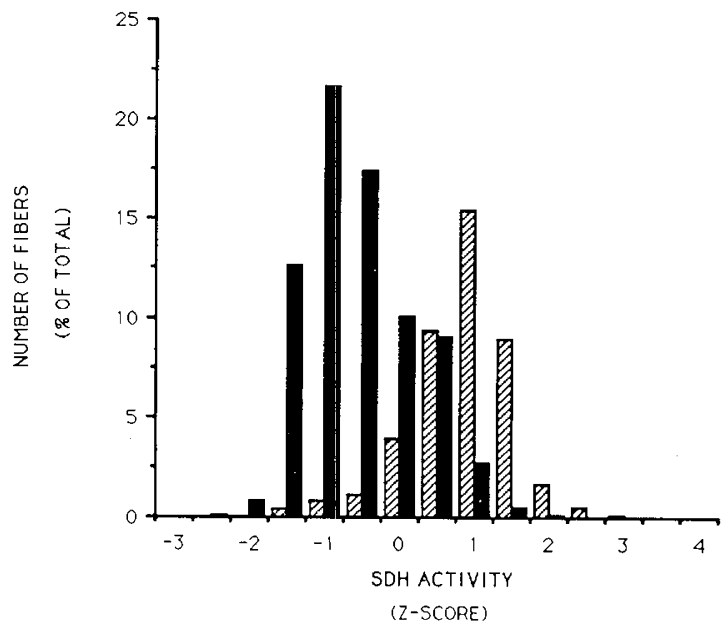

Fig. 3. Histograms showing the distributions of relative SDH activities (normalized for Z-scores) of type I (cross-hatched) and type II (filled bars) muscle fibers from diaphragms at different ages: A, 1st wk; B, 2nd wk; C, 3rd wk; D, 6th wk; and E, adult. Note that the separation of SDH activities of type I and II fiber populations occurred only by the 6th postnatal wk. 


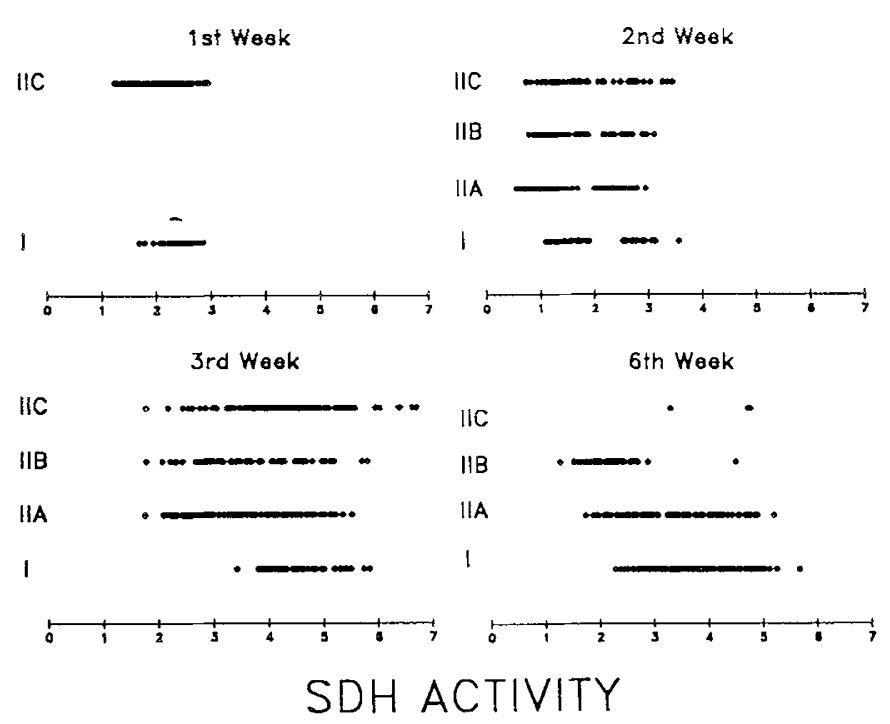

Fig. 4. Scatter plots showing the distribution of SDH activities ( $\mathrm{mmol}$ fumarate/L tissue/min) for different fiber types from diaphragms of animals representing cach postnatal age. Note that the SDH activities of type IIB fibers were lower than those of type I and IIA fibers only by the 6th postnatal wk.

This difference in size was due to the larger CSA of type IIB fibers $(p<0.01)$, inasmuch as the CSA of type IIA fibers was generally comparable to that of type I fibers (Fig. 8). Throughout the first $6 \mathrm{wk}$ of postnatal development, there were no differences in CSA among type IIA, IIB, or IIC fibers (Fig. 8).

The distributions of CSA (Z-scores) for type I and II fibers were unimodal at each age. There was considerable overlap in the distribution of CSA between fiber types (Fig. 9). Even by the 6th postnatal wk, the CSA of type IIB fibers were comparable to those of type $I$ and IIA fibers (Figs. 8 and 9).

The CV of fiber CSA showed very little change during postnatal development (e.g. 1st wk $C V=40.4 \pm 4.3 \%$ versus 6 th $\mathrm{wk}$ $\mathrm{CV}=39.8 \pm 3.3 \%$ ). This compared to a $\mathrm{CV}$ of $47.3 \pm 9.9 \%$ for CSA among adult diaphragm fibers. The IQR of fiber CSA increased progressively during postnatal development, reflecting the progressive growth of fibers (e.g. 1st wk IQR $=132.9 \pm 29.5$ $\mu \mathrm{m}^{2}$ versus 6 th wk IQR $\left.=352.1 \pm 60.3 \mu \mathrm{m}^{2} ; p<0.01\right)$. The IQR of adult fiber CSA was $945.7 \pm 100.5 \mu \mathrm{m}^{2}$.

Relationship between fiber CSA and SDH activity. In the adult diaphragm, fibers with the lower SDH activities generally have larger CSA $(6,7)$. During postnatal development, this inverse relationship between fiber size and SDH activity was not apparent.

\section{DISCUSSION}

Lack of objective basis for subclassifying diaphragm muscle fibers based on SDH activity. In this study, we examined changes in the distributions of fiber CSA and SDH activities during postnatal development of the cat diaphragm. At each age, the distribution of both CSA and SDH activities in the diaphragm were unimodal for both type I and II fibers. The unimodal distribution of SDH activities among type II fibers in the developing diaphragm is similar to that which we previously observed in the adult muscle $(6,7)$. These results indicate that differences in oxidative capacity cannot be used to subclassify type II fibers at any age in the cat diaphragm. Similar unimodal distributions of SDH activities have been observed for type II fibers in the rat, rabbit, and hamster diaphragms (24). The lack of an objective criterion for subclassifying type II fibers may explain in part why previous studies using oxidative capacity as the basis for fiber typing during development have yielded conflicting results (e.g. $1-3)$.

Subclassification of diaphragm muscle fibers based on ATPase staining intensity. It was possible to subclassify type II fibers in the developing cat diaphragm based on quantitative differences in staining intensities for myofibrillar ATPase after alkaline and acid preincubations (compare 4,5 ). It was observed that most fibers in the neonatal (1st wk) cat diaphragm would be histochemically classified as type IIC. It is likely, however, that these type IIC fibers are actually composed of a mixture of Mhc isoforms (25-27). During development, the differentiation of adult type II fibers (i.e. expressing predominantly a single Mhc isoform) involves the sequential expression of an embryonic, a neonatal, and finally the adult isoform of fast (type II) Mhc (25). In addition, most of these future adult type II fibers also express the slow (type 1) Mhc. At some point in postnatal development, the expression of the slow Mhc in type II fibers is suppressed. In contrast, the differentiation of adult type I fibers involves the continued expression of the slow Mhc (25). Initially, the future adult type I fibers may also express more than a single Mhc isoform, (e.g. both the slow and embryonic isoforms). The expression or suppression of the slow Mhe is modulated by the pattern of innervation during development and the hormonal environment (e.g. thyroid hormone) (17). Therefore, some of the type IIC fibers in the neonatal diaphragm are apparently destined to become adult type II fibers whereas other type IIC fibers will eventually differentiate into adult type I fibers. The final proportions can vary depending on the pattern of innervation established during early development, the process of synapse elimination and the changing hormonal environment.

SDH activities of different diaphragm muscle fiber types. Whether the type IIC fibers destined to become adult type I fibers vary in their SDH activity (e.g. higher activity) from those destined to become adult type II fibers could not be clearly ascertained in our present study. However, we observed that, during early postnatal development, no clear differences were present in the distribution of SDH activities among type IIA, IIB, or IIC fibers. Based on the similarity in the relative distribution of SDH activities among fibers at each age, it would appear unlikely that the developmental transitions of fibers from

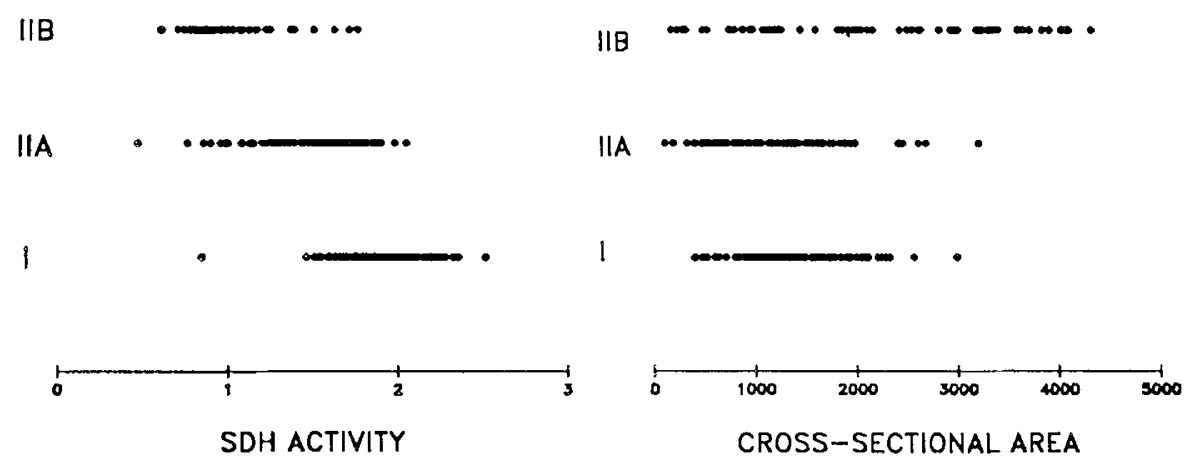

Fig. 5. Scatter plots showing the distribution of SDH activities ( $\mathrm{mmol}$ fumarate/L tissue/min) and CSA ( $\mu \mathrm{m}^{2}$ ) of different fiber types in an adult diaphragm. Note that the type IIB fibers were generally larger and had higher SDH activities than either type I or IIA fibers. 


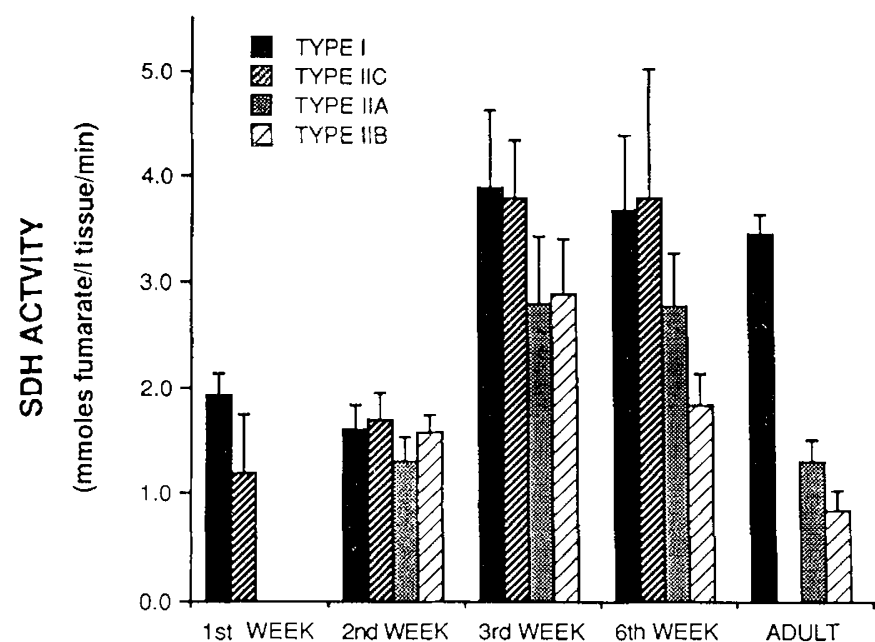

Fig. 6. Postnatal changes in the mean $( \pm S D) S D H$ activities of type I. IIA. IIB, and IIC fibers. The SDH activities of both type I and II fibers were relatively low during the first $2 \mathrm{wk}$ of postnatal development. From the 3rd to the 6th wk of age, SDH activities of all fiber types were elevated. Thereafter, the SDH activities of both type I and II fibers decreased to adult values. Note that differences between type I and II fibers were significant $(p<0.01)$ only after the 3 rd postnatal wk.

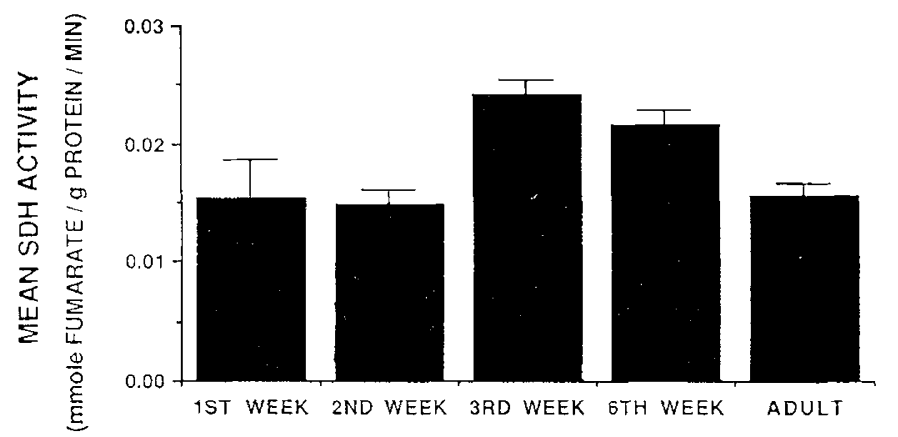

Fig. 7. The mean $( \pm \mathrm{SD}) \mathrm{SDH}$ activity of whole muscle homogenates of the diaphragm showed the same developmental trend as the changes in SDH activities of single fibers.

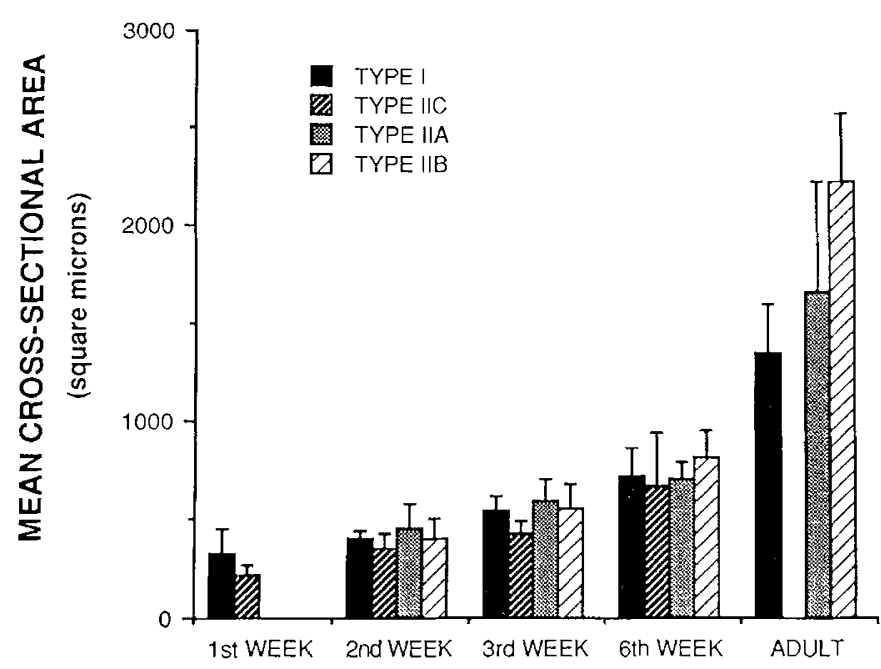

Fig. 8. The mean $( \pm \mathrm{SD}) \mathrm{CSA}$ of all muscle fiber types of the diaphragm increased progressively during postnatal development. Type IIB fibers displayed the greatest change in cross-sectional area.

type IIC to I could be distinguished based on differences in fiber SDH activity.

In the adult diaphragm, the mean SDH activity of type IIA fibers was significantly higher than that of IIB fibers. It should

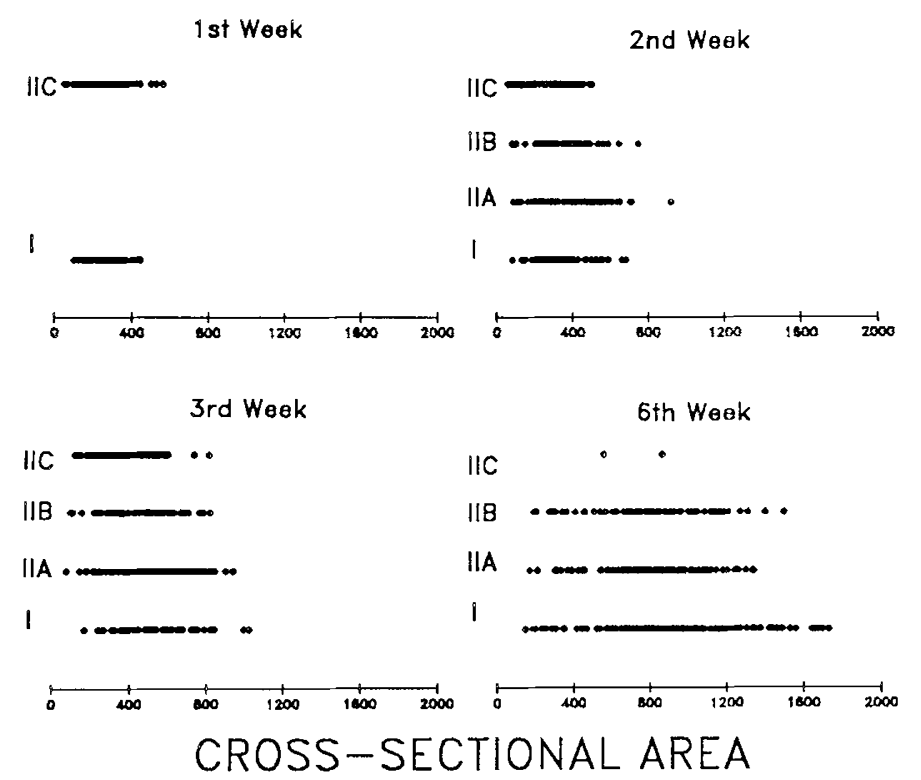

Fig. 9. Scatter plots showing the distribution of CSA of different fiber types from diaphragms of animals representing different postnatal ages. Note that the size of type IIB fibers was comparable to that of type I and IIA fibers throughout the $6 \mathrm{wk}$ of postnatal development.

be noted, however, that cat diaphragm fibers histochemically classified as type IIB may actually contain primarily a IIX Mhc isoform (28-32). Based on electrophoretic migration patterns of Mhe isoforms in an SDS-polyacrylamide gel, we found bands located in positions corresponding to the rat type I, IIA, and IIX Mhe isoforms, but the band corresponding to the IIB isoform in the rat was absent (unpublished observations). These results suggest that in the cat diaphragm, as in the rat, fibers classified histochemically as type IIB may actually contain predominantly the IIX Mhc isoform. Whether this would influence the distribution of fiber SDH activities is unclear. Based on a subjective evaluation of staining intensity for $\mathrm{SDH}$, it has been reported that, in the rat diaphragm, fibers containing predominantly the IIX Mhc isoform have an oxidative capacity that is intermediate between IIA and IIB fibers (31). Therefore, it is possible that the unimodal distribution of SDH activities among type II fibers in the cat diaphragm reflects the absence of fibers containing the IIB Mhc isoform (i.e. low oxidative fibers).

Postnatal changes in diaphragm muscle fiber type proportions. During the first 6-wk after birth, there was a progressive increase in the proportion of type I fibers in the diaphragm. A similar developmental trend in fiber type proportions has also been described for other mixed skeletal muscles $(13-17,24)$. However, the proportions of type I and II fibers in the diaphragm can vary across species. For example, in contrast to the neonatal cat diaphragm, where only about $10 \%$ of all fibers were type 1 , the neonatal human $(1,2)$ and baboon (3) diaphragms have a greater proportion of type I fibers (approximately 25 and $40 \%$, respectively). In the human diaphragm, Keens et al. $(1,2)$ found that, as in the cat, the proportion of type I fibers increased progressively during postnatal development, from $25 \%$ at birth to about $55 \%$ in the adult. In contrast, in the baboon diaphragm, Maxwell et al. (3) reported that the proportion of type I fibers did not increase during postnatal development, being approximately $40 \%$ in both neonates and adults.

Postnatal changes in diaphragm muscle fiber SDH activity. The SDH activities of diaphragm muscle fibers in the cat were initially low during the first 2 postnatal wk, then increased rapidly to reach their highest values by $21 \mathrm{~d}$ of age $(4,24)$. This increase in fiber SDH activity during early postnatal development is similar, in some respects, to that reported by Smith et al. (9) for rat diaphragm fibers. However, these investigators reported only a $10-20 \%$ increase in SDH activity of rat diaphragm fibers during 
the first 3 postnatal wk compared to a 2 -fold increase in the SDH activity of cat diaphragm fibers during the same postnatal period. Moreover, Smith et al. (9) reported that the SDH activities of rat diaphragm fibers plateaued such that the SDH activities of adult diaphragm fibers were higher than those of neonatal fibers. In the cat diaphragm, SDH activities of adult fibers were lower than those of fibers at 3 and $6 \mathrm{wk}$ of age. These varying results could be attributed to species differences. However, in a recent study in rats, we found a 3 -fold increase in the SDH activities of diaphragm fibers during the first $3 \mathrm{wk}$ of postnatal development (unpublished observations). Furthermore, as in the cat diaphragm, the SDH activities of fibers in the adult rat diaphragm were comparable to those of neonatal fibers and lower than those of fibers at $3 \mathrm{wk}$ of age (i.e. SDH activities did not plateau). Thus, it would appear that species differences cannot account for the differences between our results and those of Smith et al. (9). It is more likely that our varying results can be attributed to significant differences in the technique used to quantify fiber SDH activity (see ref. 4 for discussion of these differences).

Relationship between diaphragm muscle fiber size and SDH activity. The postnatal changes in fiber SDH activity were not related to changes in fiber size. In the adult cat diaphragm, there is a significant inverse correlation between fiber CSA and SDH activity $(6,7)$. Such an inverse correlation between the size and oxidative capacity of diaphragm fibers was not apparent throughout the first 6 wk of postnatal development. It is possible that the inverse relationship between fiber size and oxidative capacity in the adult diaphragm relates to the fatigue resistance of the muscle units to which fibers belong (33). Fibers comprising more fatigable fast-twitch muscle units are generally larger and have lower SDH activities than those fibers belonging to more fatigue resistant (slow- and fast-twitch) units. This correspondence to unit fatigue resistance may be related to the ability of fibers to extract oxygen from surrounding capillaries. In larger fibers, diffusion distances from capillaries to intracellular mitochondria would be increased. In agreement with this possibility, we have found that capillary density (number of capillaries per fiber CSA) is higher surrounding fibers belonging to more fatigue-resistant muscle units in the adult cat diaphragm (33). The lack of a relationship between fiber size and SDH activity early in development may be due to the much smaller size of developing diaphragm fibers, i.e. no significant diffusion gradient for any fiber. However, the absence of such a relationship between fiber size and oxidative capacity might also reflect the lack of differentiation of adult muscle units with varying fatigue resistance.

Postnatal changes in diaphragm innervation and recruitment. In the cat diaphragm, polyneuronal innervation of muscle fibers disappears only after the $3 \mathrm{rd}$ postnatal wk (24). Only then does the influence of specific innervation of fibers present itself. It is likely that functional differences in muscle unit contractile and fatigue properties develop only gradually after the disappearance of polyneuronal innervation and the emergence of significant differences in the activation history of motor units. It has been demonstrated that muscle fibers composing adult motor units display very similar metabolic capacities, most likely because of their common activation history. In the adult cat diaphragm, we estimated that the forces required by normal ventilation represented only $12 \%$ of the maximum force generating capacity of the muscle (34). These ventilatory forces could be accomplished by the recruitment of only fatigue resistant motor units. Thus, a large fraction of the diaphragm motor unit pool remains inactive during ventilatory behaviors. In this situation, a wide range of metabolic capacities among diaphragm muscle fibers would be expected. During early postnatal development, it is possible that a much larger fraction of the diaphragm is utilized to accomplish normal ventilatory behaviors. In this situation, more diaphragm muscle fibers would share a common activation history and, thus, less variance in metabolic capacity among fibers would be expected. The observation of an increase in the IQR of fiber $\mathrm{SDH}$ activities during the $3 \mathrm{rd}$ postnatal wk corresponds with the disappearance of polyneuronal innervation. Similarly, the increase in the $\mathrm{CV}$ of $\mathrm{SDH}$ activities by the 6th postnatal wk might be explained by the emergence of differences in the activation history of diaphragm motor units.

Postnatal changes in diaphragm fatigue resistance. Maxwell et al. (3) reported that the baboon diaphragm was very resistant to fatigue during early postnatal development. These authors attributed the fatigue resistance of the neonatal diaphragm to the high oxidative capacity of diaphragm muscle fibers. In a separate study (24), we also found that the neonatal cat diaphragm is fatigue resistant and that during subsequent postnatal development it becomes progressively more fatigable. However, the postnatal changes in fiber oxidative capacity noted in our present study do not correlate with changes in diaphragm fatigue resistance. The changes in diaphragm fatigue resistance do correlate, to some extent, with the postnatal changes in the size of diaphragm fibers.

It is possible that the postnatal changes in diaphragm fiber $\mathrm{SDH}$ activities are related to the changing energy demands for muscle contraction. As in other muscles, the contraction time of the neonatal cat diaphragm is considerably slower than that of the adult muscle, despite the presence of a much higher proportion of putative type II (fast-twitch) fibers (24). Slower-contracting muscle fibers are significantly more energy efficient than faster-contracting fibers (35). This difference in energy efficiency might account for both the higher fatigue resistance of the neonatal diaphragm and its lower oxidative capacity.

Summary. In summary, the results of our study indicate that differences in oxidative capacity cannot be used to subclassify type II fibers in the cat diaphragm at any age. The distributions of SDH activities were unimodal for both type I and II fibers. Among type II fibers, the SDH activities of IIA, IIB, and IIC fibers displayed considerable overlap. Changes in fiber SDH activity during postnatal development were not progressive. Fiber SDH activities were uniformly low at birth. By 3 wk of age, fiber SDH activities were the highest of any age. The CSA of diaphragm fibers increased progressively during postnatal development. The increase in diaphragm fiber size paralleled the increase in body weight. Unlike in the adult, there was no inverse relationship between the size and SDH activity of diaphragm fibers at any time during the first $6 \mathrm{wk}$ of postnatal development.

Acknowledgments. The authors would like to ackowledge the valuable contributions of Tracey Cheung in these studies. The authors also thank Drs. Robert Guthrie and William LaFramboise for contributions in preliminary studies assessing myosin heavy chain isoform expression in the cat diaphragm.

\section{REFERENCES}

1. Keens TG, Bryan AC, Levison H, Ianuzzo CD 1978 Developmental pattern of muscle fiber types in human ventilatory muscles. J Appl Physiol 44:909913

2. Keens TG, Ianuzzo CD 1979 Development of fatigue-resistant muscle fibers in human ventilatory muscles. Am Rev Respir Dis 2:139-141

3. Maxwell LC, McCarter RJM, Kuehl TJ, Robotham JL 1983 Development of histochemical and functional properties of baboon respiratory muscles. J Appl Physiol 54:551-561

4. Sieck GC, Cheung TS, Blanco CE 1991 Diaphragm capillarity and oxidative capacity during postnatal development. J Appl Physiol 70:103-111

5. Sieck GC, Fournier M, Enad JG 1989 Fiber type composition of muscle units in the cat diaphragm. Neurosci Lett 97:29-34

6. Sieck GC, Sacks RD, Blanco CE 1987 Absence of regional differences in the size and oxidative capacity of diaphragm muscle fibers. J Appl Physiol 63:1076-1082

7. Sieck GC, Sacks RD, Blanco CE, Edgerton VR 1986 SDH activity and crosssectional area of muscle fibers in cat diaphragm. J Appl Physiol 60:1284 1292

8. Sieck GC, Roy RR, Powell P, Blanco CE, Edgerton VR, Harper RM 1983 Muscle fiber type distribution and architecture of the cat diaphragm. J Appl Physiol 55:1386-1392

9. Smith D, Green H, Thomson J, Sharratt M 1988 Oxidative potential in developing rat diaphragm, EDL, and soleus muscle fibers. Am J Physio 254:C661-C668

10. Brooke MH, Kaiser KK 1970 Muscle fiber types: how many and what kind? Arch Neurol 23:369-379 
11. Brooke MH, Williamson E, Kaiser KK 1971 The behavior of four fiber types in developing and reinnervated muscle. Arch Neurol 25:360-366

12. Peter JB, Barnard RJ, Edgerton VR, Gillespie CA, Stempel KE 1972 Metabolic profiles of thrce fiber types of skeletal muscle in guinea pigs and rabbits. Biochemistry 11:2627-2634

13. Cooper CC, Cassens RG, Kastenschmidt LL, Briskey JE 1970 Histochemical characterization of muscle differentiation. Dev Biol 23:169-184

14. Dubowitz V 1965 Enzyme histochemistry of skeletal muscle. I. Developing animal muscle. J Neurol Neurosurg Psychiatry 28:516-519

15. Dubowitz V 1965 Enzyme histochemistry of skeletal muscle. II. Developing human muscle. J Neurol Neurosurg Psychiatry 28:519-524

16. Nystrom B 1968 Histochemistry of developing cat muscles. Acta Neurol Scand 44:405-439

17. Tomanek RJ 1975 A histochemical study of postnatal differentiation of skeleta muscle with reference to functional overload. Dev Biol 42:305-314

18. Blanco CE, Sieck GC, Edgerton VR 1986 Quantitative histochemical determination of succinic dehydrogenase activity in skeletal muscle fibres. Histochem J 20:230-243

19. Blanco CE, Sieck GC 1987 Comparison of succinate dehydrogenase activity between the diaphragm and medial gastrocnemius muscles of the rat. In: Sieck GC, Cameron WE, Gandevia SC (eds) Respiratory Muscles and Their Neuromotor Control. Alan R Liss, Inc, New York pp 28 I-289

20. Pennington R 1961 Biochemistry of dystrophic muscle: mitochondrial succinate-tetrazolium reductase and adenosine triphosphatase. Biochem $\mathrm{J}$ 80:649 654

21. Bradford MM 1976 Rapid and sensitive method for quantification of microgram quantities of protein utilizing the principle of protein-dye binding. Anal Biochem 72:248-252

22. Afifi AA, Azen SP 1979 Statistical Analysis: A Computer-Oriented Approach. Academic Press, New York

23. Dixon WJ 1985 BMDP Statistical Software. University of California Press, Los Angeles
24. Sicck GC, Fournier M 1991 Developmental aspects of diaphragm muscle cells: structural and functional organization. In: Haddad GG. Farber JP (eds) Developmental Neurobiology of Breathing. Marcel Dekker, New York, pp $375-428$

25. Kelly AM 1983 Emergence of muscle specialization. In: Handbook of Physiology, Section 10. Skeletal Muscie. American Physiological Society, Bethesda, pp 507-537

26. LaFramboise WA, Daood MJ, Brozanski BS, Guthrie RD, Ontell M 1989 Myosin heavy chain transitions in the developing diaphragm. Am Rev Respir Dis 139:A166(abstr)

27. Reiser PJ, Moss RL, Giulian GG, Greaser ML 1985 Shortening velocity and myosin heavy chains of developing rabbit muscle fibers. I Biol Chem 260:14403-14405

28. Bar A, Pette D 1988 Three fast myosin heavy chains in adult rat skeletal muscle. FEBS Lett 235:153-155

29. LaFramboise WA, Daood MJ, Guthrie RD, Moretti P, Schiaffino S, Ontell M 1990 Electrophoretic separation and immunological identification of type $2 \mathrm{X}$ myosin heavy chain in rat skeletal muscle. Biochim Biophys Acta 1035:109-112

30. Schiaffino S, Ausoni S, Gorza L, Saggin L, Gundersen K, Lomo T 1988 Myosin heavy chain isoforms and velocity of shortening of type 2 skeletal muscle fibers. Acta Physiol Scand 134:575-576

31. Schiaffino S, Gorza L, Sartore S, Saggin L, Ausoni S, Vianello M, Gundersen K, Lomo T 1989 Three myosin heavy chain isoforms in type 2 skelctal muscle fibers. J Muscle Res Cell Motil 10:197-205

32. Termin A, Staron RS, Pette D 1989 Myosin heavy chain isoforms in histochemically defined fiber types of rat muscle. Histochemistry 92:453-457

33. Enad JG, Fournier M, Sieck GC 1989 Oxidative capacity and capillary density of diaphragm motor units. J Appl Physiol 67:620-627

34. Sieck GC, Fournier M 1989 Diaphragm motor unit recruitment during ventilatory and non-ventilatory behaviors. J Appl Physiol 66:2539-2545

35. Crow MT, Kushmerick MJ 1982 Chemical energies of slow- and fast-twitch muscles of the mouse. J Gen Physiol 79:147-166 